\title{
Surviving ischemia: adaptive responses mediated by hypoxia-inducible factor 1
}

\author{
Gregg L. Semenza \\ Institute of Genetic Medicine and Departments of Pediatrics and Medicine, The Johns Hopkins University School of Medicine, \\ Baltimore, Maryland, USA \\ Address correspondence to: Gregg L. Semenza, Johns Hopkins Hospital, CMSC-1004, 600 North Wolfe Street, \\ Baltimore, Maryland 21287-3914, USA. Phone: (410) 955-1619; Fax: (410) 955-0484; E-mail: gsemenza@jhmi.edu.
}

While the other Perspectives in this series provide readers with state-of-the-art overviews of responses to ischemia on an organ-by-organ basis, I focus here on a single transcription factor, hypoxia-inducible factor 1 (HIF-1), and its role in the physiologic responses to hypoxia and ischemia. Whether in the brain, heart, kidneys, lungs, or muscle, HIF-1 is essential for ischemiainduced angiogenesis, as described by Isner (this Perspective series, ref. 1), and it may also be a critical mediator of late-phase preconditioning, as described by Williams and Benjamin (this Perspective series, ref. 2). For these reasons, HIF-1 and the genes under its control may represent novel therapeutic targets for ameliorating the effects of ischemia in a variety of clinical settings.

Molecular biology of HIF-1

HIF-1 is a heterodimeric transcription factor consisting of HIF- $1 \alpha$ and HIF- $1 \beta$ subunits. The amino-terminal half of each subunit contains basic helix-loophelix (bHLH) and PAS motifs that are required for dimerization and DNA binding. Other domains in the carboxyl-terminal half of HIF- $1 \alpha$ mediate hypoxiainducible nuclear localization, protein stabilization, and transactivation (reviewed in ref. 3). Thus, whereas HIF-1 $\beta$ (also known as the aryl hydrocarbon receptor nuclear translocator) can heterodimerize with other proteins that contain a bHLH-PAS domain, HIF- $1 \alpha$ is the specific and oxygen-regulated subunit of HIF-1. Increased HIF-1 activity leads to transcription of genes that are expressed in most cell types, such as those encoding glucose transporters, glycolytic enzymes, and VEGF, as well as genes that are expressed in a cell type-specific manner, such as erythropoietin, inducible nitric oxide synthase (NOS2), and insulin-like growth factor 2 (IGF-2). Although other transcription factors, such as AP-1, EGR-1, NF- $\kappa B$, and NF-IL-6 mediate the hypoxia-inducible expression of specific genes in specific cell types (reviewed in ref. 4), HIF-1 appears to be unique with respect to its function as a global regulator of oxygen homeostasis. HIF-1-regulated genes contain a hypoxia response element (HRE), a cis-acting transcriptional-regulatory element that includes one or more binding sites for HIF-1. In the case of the human VEGF gene, an HRE located $1 \mathrm{~kb} 5^{\prime}$ to the start site mediates hypoxia- and HIF-1-dependent transcription (5). The crucial role of this interaction has been demonstrated in HIF- $1 \alpha$-null mouse embryonic stem (ES) cells, where basal VEGF mRNA expression is reduced and does not increase in response to hypoxia (6-8). HIF- $1 \alpha$ is required for normal vascularization, development, and survival of mouse embryos as well as for physiologic responses to chronic hypoxia in adult mice (6-10).

The variable angiogenic responses of ischemic tissues

Ischemia and infarction occur when tissue perfusion is insufficient to meet metabolic demands. In animal models of coronary artery occlusion, myocardial ischemia induces VEGF expression and collateral blood vessel development $(11,12)$. In humans, many patients with coronary artery stenosis fail to develop collateral vessels. Because there is an inverse correlation between infarct size and collateral blood flow $(13,14)$, such individual variation in adaptive responses to ischemia may determine the risk and severity of myocardial infarction. I will describe the angiogenic response potential as manifested in an animal model, discuss molecular mechanisms underlying the limited angiogenic response in humans, and consider strategies to circumvent these limitations.

To study the effects of hypoxia in the developing heart in vivo, near-term fetal sheep were subjected to a daily partial exchange transfusion in which blood was replaced with saline (15). Under these conditions, the hematocrit and arterial oxygen content are gradually reduced to onethird of normal levels within one week. Concomitantly, cardiac output increases by $50 \%$ and the heart/body weight ratio increases by $30 \%$. To maintain myocardial oxygenation under these circumstances, myocardial blood flow increases fivefold. Capillary density and minimal capillary diameter are significantly increased and intercapillary distance is decreased in the hearts of anemic compared with control fetuses (15). Underlying these differences are three- to fourfold increases in the expression of VEGF protein and mRNA, as well as HIF- $1 \alpha$ pro- 
tein. In these anemic sheep fetuses, increased cardiac output provides systemic compensation for decreased oxygen-carrying capacity. The increased cardiac work and mass increase myocardial oxygen consumption, thus providing a hypoxic stimulus for increased HIF- $1 \alpha$ expression and HIF-1-mediated VEGF gene transcription. Temporo-spatial correlations between HIF- $1 \alpha$ protein and VEGF mRNA expression have also been demonstrated in the context of developmental and ischemic vascularization of the mouse retina (16).

Why does this response pathway fail to provide sufficient neovascularization in patients with coronary artery disease to prevent ischemia? One major difference is that the fetal sheep heart is a growing organ that is actively engaged in angiogenesis. Although this suggests differences in angiogenic responses as a function of developmental stage, aging in general may be an important factor. The absence of collateralization in some patients with symptomatic coronary artery disease may reflect impaired ability to produce VEGF. Ischemia-induced VEGF expression is likely to be influenced by a combination of genetic and environmental factors. Recent studies described below suggest that both aging and individual variability in responses to ischemia may influence the outcome of atherosclerotic vascular disease.

Collateral vessel development following femoral artery ligation is significantly reduced in older mice and rabbits (17). The impairment in vascularization is multifactorial, as older animals exhibit impaired VEGF production, vascular endothelial cell dysfunction, and reduced lymphocytic infiltration of ischemic tissues. Aortic smooth muscle cells from old rabbits manifest impaired hypoxia-induced VEGF expression due to a reduction in HIF-1 DNA-binding activity (18). HIF-1 DNA-binding activity is also reduced in brain, kidney, liver, and lung tissue from old versus young mice subjected to hypoxia (19).

These results do not address why individuals of similar age and degree of coronary occlusion vary in the extent of collateralization. Peripheral blood mononuclear cells isolated from patients with no angiographic evidence of coronary collateralization manifest significantly reduced induction of VEGF mRNA in response to hypoxia compared with cells from individuals with collateral development (20). This difference is maintained after analysis of multiple covariates. Thus, genetic or environmental factors or both may determine the physiological response to ischemia in each individual. VEGF production may be modulated via changes in the expression or activity of HIF-1. If so, it may be possible to identify individuals with suboptimal ischemiainduced VEGF expression; such individuals in particular may be candidates for therapeutic angiogenesis.

Clinical trials involving VEGF protein administration or VEGF gene therapy are underway (reviewed in ref. 21). It is unclear whether VEGF alone is sufficient to induce development of normally-functioning collateral vessels that will correct perfusion defects in ischemic myocardium. Whereas transgenic mice overexpressing VEGF exhibit excessive vascular permeability, mice overexpressing both VEGF and angiopoietin-1 manifest normal vascular permeability (22). HIF- $1 \alpha$ gene therapy may have the advantage of inducing the expression not only of VEGF, but also of other hypoxiainduced angiogenic or survival factors (such as angiopoietin-2 and IGF-2) and their receptors on endothelial cells (such as FLT-1). In a recent study, HIF$1 \alpha$ gene therapy was as effective as VEGF in stimulating therapeutic angiogenesis in a rabbit hindlimb ischemia model (23). Furthermore, transgenic mice expressing HIF- $1 \alpha$ in the skin demonstrated increased vascular density, as in the case of VEGF transgenic mice, but whereas VEGF transgenic mice demonstrate increased capillary leakage in response to an inflammatory stimulus, in HIF- $1 \alpha$ transgenic mice there was actually decreased capillary leakage compared with nontransgenic littermates (J. Arbeit, personal communication). Whether this property is due to HIF-1-mediated expression of angiopoietin-2, which is known to be induced by hypoxia (24), remains to be established.

\section{Cerebral ischemia}

Following permanent middle cerebral artery occlusion in rats, there is a temporal and spatial co-induction of HIF- $1 \alpha$ mRNA with mRNAs encoding glucose transporter 1 , the glycolytic enzymes aldolase A, lactate dehydrogenase A, phosphofructokinase L, and pyruvate kinase $M$, and $\operatorname{VEGF}(25,26)$, all of which are encoded by HIF-1 target genes $(7,8)$. Expression of these mRNAs is localized to the penumbra (viable tissue surrounding the infarction), suggesting that HIF1-mediated induction of angiogenesis and glycolytic metabolism may be important for the survival of these cells $(25,26)$. In contrast, when a truncated form of HIF- $1 \alpha$ that has dominant-negative effects (5) is expressed in cultured neuronal cells, delayed cell death in response to oxygen and glucose deprivation is reduced (27). The protective effect of dominant-negative HIF- $1 \alpha$ expression is not observed in p53-null neurons, consistent with observations of HIF$1 \alpha$-induced p53-mediated death of ES cells subjected to oxygen and glucose deprivation (6). Severity of hypoxia, induction of $\mathrm{p} 53$, and apoptosis are correlated in cultured neurons (28). NOS2, the product of another known HIF-1 target gene (29), also has a proapoptotic role during cerebral ischemia $(30,31)$. In rats subjected to hypoxia and unilateral permanent middle cerebral artery occlusion, HIF- $1 \alpha$ expression is increased throughout the contralateral cortex whereas, on the ipsilateral side, parenchymal expression is decreased and there is a striking increase in HIF- $1 \alpha$ expression in medium-sized cortical feeder vessels (32). Further studies are necessary to establish the conse- 
quences of HIF-1 expression in ischemic glial, neuronal, inflammatory, and vascular cells in vivo and the role of HIF-1 in promoting or preventing cerebral infarction following arterial occlusion.

\section{Late-phase preconditioning}

Exposure of 1 -week-old rats to hypoxia $\left(8 \% \mathrm{O}_{2}\right)$ for 3 hours protects against cerebral infarction in animals subjected to combined hypoxia and ischemia (left common carotid artery ligation) 24 hours later (33). The hypoxic preconditioning is associated with increased HIF- $1 \alpha$ expression throughout the brain (32). Expression of HIF- $1 \alpha$ mRNA and protein is also induced in the brains of neonatal rats following intraperitoneal injection of cobalt chloride or desferrioxamine, and, like hypoxia, prior cobalt or desferrioxamine administration provides protection against cerebral infarction following hypoxia and ischemia. Furthermore, the rank order of potency (hypoxia > cobalt > desferrioxamine) is similar for induction of HIF- $1 \alpha$ expression and for cerebral protection (32).

HIF- $1 \alpha$ expression is also likely to be involved in cardiac preconditioning, since NOS2 expression is essential for late-phase (delayed) cardiac preconditioning (34), and an intact HIF-1 DNA-binding site in the NOS2 promoter is required for its transcriptional induction in hypoxic myocardial cells (29). Nitric oxide (NO) appears to play a dual role by acting as both a trigger and a mediator of delayed preconditioning (35). NO donors induce HIF-1 $\alpha$ expression and HIF-1 activity in cultured cells under nonhypoxic conditions (36). Thus, an initial NO signal may act as a trigger to induce HIF-1 activity, leading to subsequent expression of NOS2 and more production of NO, which then mediates protective effects.

\section{Conclusions}

Recent studies have demonstrated the essential role of HIF-1 in hypoxia-induced, VEGF-mediated angiogenesis and suggest that HIF-1 mediates hypoxia-induced preconditioning in the brain, heart, and other organs. HIF-1 may also contribute both to the survival of hypoxic-ischemic cells and, paradoxically, to the death of cells that are unable to adapt to hypoxia or ischemia. In addition to promoting p53-mediated apoptosis as described above, HIF-1 has recently been shown to transactivate the proapoptotic NIP3 gene under hypoxic conditions (37).

In tissue culture, HIF-1 activity is induced by hypoxia to a relatively similar degree in most cells. Nevertheless, individual cell types display markedly different optimal oxygen concentrations for growth and varying resistance to hypoxia-induced apoptosis. Hence, it appears that other cell type-specific factors (which may include related factors, such as HIF- $2 \alpha$ and HIF- $3 \alpha$, and unrelated factors, such as AP-1, EGR-1, and NF- $\kappa$ B) are required to interpret the hypoxic signal that is trans- mitted to the nucleus by HIF-1. Thus, even when one focuses on the role of a single transcription factor in surviving ischemia, one cannot help but be struck by the complexity of biochemical and physiological interactions. At present, these interactions are poorly understood. Yet, as seen throughout this Perspective series, rapid and dramatic progress has been made in understanding tissue responses to ischemia, an accomplishment that justifies optimism regarding the application of this basic research to clinical practice.

\section{Acknowledgments}

Work in the author's laboratory is supported by grants from the NIH (R01-DK-39869 and R01-HL-55338). G.L. Semenza is a consultant to Genzyme Corporation which has been granted a license by Johns Hopkins University for HIF- $1 \alpha$ gene therapy of cardiovascular disorders. The terms of these arrangements are managed by the University in accordance with its conflictof-interest policies.

1. Isner, J.M. 2000. Tissue responses to ischemia: local and remote responses for preserving perfusion of ischemic muscle. J. Clin. Invest. 106:615-619.

2. Williams, R.S., and Benjamin, I.J. 2000. Protective responses in the ischemic myocardium. J. Clin. Invest. 106:813-818.

3. Semenza, G.L. 2000. HIF-1: mediator of physiological and pathophysiological responses to hypoxia. J. Appl. Physiol. 88:1474-1480.

4. Semenza, G.L. 1999. Regulation of mammalian $\mathrm{O}_{2}$ homeostasis by hypoxia-inducible factor 1. Annu. Rev. Cell Dev. Biol. 15:551-578.

5. Forsythe, J.A., et al. 1996. Activation of vascular endothelial growth factor gene transcription by hypoxia-inducible factor $1 . \mathrm{Mol}$. Cell. Biol. 16:4604-4613.

6. Carmeliet, P., et al. 1998. Role of HIF-1 $\alpha$ in hypoxia-mediated apoptosis, cell proliferation, and tumour angiogenesis. Nature. 394:485-490.

7. Iyer, N.V., et al. 1998. Cellular and developmental control of $\mathrm{O}_{2}$ homeostasis by hypoxia-inducible factor $1 \alpha$. Genes Dev. 12:149-162.

8. Ryan, H.E., Lo, J., and Johnson, R.S. 1998. HIF-1 $\alpha$ is required for solid tumor formation and embryonic vascularization. EMBOJ. 17:3005-3015.

9. Kotch, L.E., Iyer, N.V., Laughner, E., and Semenza, G.L. 1999. Defective vascularization of HIF-1 $\alpha$-null embryos is not associated with VEGF deficiency but with mesenchymal cell death. Dev. Biol. 209:254-267.

10. Yu, A.Y., et al. 1999. Impaired physiological responses to chronic hypoxia in mice partially deficient for hypoxia-inducible factor $1 \alpha$. J. Clin. Invest. 103:691-696.

11. Banai, S., et al. 1994. Upregulation of vascular endothelial growth factor expression induced by myocardial ischemia: implication for coronary angiogenesis. Cardiovasc. Res. 28:1176-1179.

12. White, F.C., Carroll, S.M., Magnet, A., and Bloor, C.M. 1992. Coronary collateral circulation in swine after coronary artery occlusion. Circ. Res. 71:1490-1500.

13. Habib, G.B., et al. 1991. Influence of coronary collaterals on myocardial infarct size in humans: results of phase I thrombolysis in myocardial infarction (TIMI) trial. Circulation. 83:739-746.

14. Sabia, P.J., et al. 1992. An association between collateral blood flow and myocardial viability in patients with recent myocardial infarction. $N$. Engl. J. Med. 327:1825-1831.

15. Martin, C., et al. 1998. Cardiac hypertrophy in chronically anemic sheep: increased vascularization is associated with increased myocardial expression of vascular endothelial growth factor and hypoxia-inducible factor 1. Am. J. Obstet. Gynecol. 178:527-534.

16. Ozaki, H., et al. 1999. Hypoxia-inducible factor $1 \alpha$ is increased in ischemic retina: temporal and spatial correlation with VEGF expression. Invest. Ophthalmol. Vis. Sci. 40:182-189.

17. Rivard, A., et al. 1999. Age-dependent impairment of angiogenesis. Circulation. 99:111-120.

18. Rivard, A., et al. 2000. Age-dependent defect in VEGF expression is associated with reduced HIF-1 activity. J. Biol. Chem. In press. 
19. Frenkel-Denkberg, G., Gershon, D., and Levy, A.P. 1999. The function of hypoxia-inducible factor 1 (HIF-1) is impaired in senescent mice. FEBS Lett. 462:341-344.

20. Schultz, A., et al. 1999. Interindividual heterogeneity in the hypoxic regulation of VEGF: significance for the development of the coronary artery collateral circulation. Circulation. 100:547-552.

21. Losordo, D.W., Vale, P.R., and Isner, J.W. 1999. Gene therapy for myocardial angiogenesis. Am. Heart J. 138:132-141.

22. Thurston, G., et al. 1999. Leakage-resistant blood vessels in mice transgenically overexpressing angiopoietin-1. Science. 286:2511-2514.

23. Vincent, K.A., et al. 2000. Angiogenesis is induced in a rabbit model of hindlimb ischemia by naked DNA encoding a HIF-1 $\alpha /$ VP-16 hybrid transcription factor. Circulation. In press.

24. Mandriota, S.J., and Pepper, M.S. 1998. Regulation of angiopoietin-2 mRNA levels in bovine microvascular endothelial cells by cytokines and hypoxia. Circ. Res. 83:852-859.

25. Bergeron, M., Yu, A.Y., Solway, K.E, Semenza, G.L., and Sharp, F.R. 1999. Induction of hypoxia-inducible factor-1 (HIF-1) and its target genes following focal ischemia in rat brain. Eur. J. Neurosci. 11:1-12.

26. Marti, H.J., et al. 2000. Hypoxia-induced vascular endothelial growth factor expression precedes neovascularization after cerebral ischemia. Am. J. Pathol. 156:965-976.

27. Halterman, M.W., Miller, C.C., and Federoff, H.J. 1999. Hypoxiainducible factor- $1 \alpha$ mediates hypoxia-induced delayed neuronal death that involves p53. J. Neurosci. 19:6818-6824.

28. Banasiak, K.J., and Haddad, G.G. 1998. Hypoxia-induced apoptosis: effect of hypoxic severity and role of $\mathrm{p} 53$ in neuronal cell death. Brain Res. 797:295-304.

29. Jung, F., Palmer, L.A., Zhou, N., and Johns, R.A. 2000. Hypoxic regula- tion of inducible nitric oxide synthase via hypoxia inducible factor- 1 in cardiac myocytes. Circ. Res. 86:319-325.

30. Hewett, S.J., Muir, J.K., Lobner, D., Symons, A., and Choi, D.W. 1996. Potentiation of oxygen-glucose deprivation-induced neuronal death after induction of iNOS. Stroke. 27:1586-1591.

31. Iadecola, C., Zhang, F., Casey, R., Clark, H.B., and Ross, M.E. 1996. Inducible nitric oxide synthase gene expression in vascular cells after transient focal cerebral ischemia. Stroke. 27:1373-1380.

32. Bergeron, M., et al. 2000. Hypoxia-inducible factor 1 (HIF-1) role in hypoxia-induced ischemic tolerance in neonatal rat brain. Ann. Neurol. In press.

33. Gidday, J.M., Fitzgibbons, J.C., Shah, A.R, and Park, T.S. 1994. Neuroprotection from ischemic brain injury by hypoxic preconditioning in the neonatal rat. Neurosci. Lett. 168:221-224.

34. Guo, Y., et al. 1999. The late phase of ischemic preconditioning is abrogated by targeted disruption of the inducible NO synthase gene. Proc. Natl. Acad. Sci. USA. 96:11507-11512.

35. Bolli, R., et al. 1997. The protective effect of late preconditioning against myocardial stunning in conscious rabbits is mediated by nitric oxide synthase: evidence that nitric oxide acts both as a trigger and as a mediator of the late phase of ischemic preconditioning. Circ. Res. 81:1094-1107.

36. Kimura, H., et al. 2000. Hypoxia response element of the human vascular endothelial growth factor gene mediates transcriptional regulation by nitric oxide: control of hypoxia-inducible factor 1 activity by nitric oxide. Blood. 95:189-197.

37. Bruick, R.K. 2000. Expression of the gene encoding the proapoptotic nip3 protein is induced by hypoxia. Proc. Natl. Acad. Sci. USA. 97:9082-9087. 